\title{
Aikaterini Véroni et Evanghélia Paraskévopoulou : deux « grandes actrices » de la scène grecque du $\mathrm{XIX}^{\mathrm{e}}$ siècle
}

Aikaterini Veroni and Evangelia Paraskevopoulou, Two Major Actresses of the Greek Scene in the 19th Century

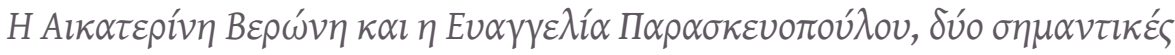

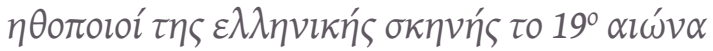

\section{Alexia Altouva}

\section{CpenEdition}

Journals

Édition électronique

URL : https://journals.openedition.org/ceb/5582

DOI : $10.4000 / c e b .5582$

ISSN : 2261-4184

\section{Éditeur}

INALCO

Édition imprimée

Date de publication : 1 mars 2015

ISBN : 978-2-85831-224-5

ISSN : 0290-7402

\section{Référence électronique}

Alexia Altouva, «Aikaterini Véroni et Evanghélia Paraskévopoulou : deux « grandes actrices » de la scène grecque du xix siècle », Cahiers balkaniques [En ligne], Hors-série | 2015, mis en ligne le 11 décembre 2015, consulté le 06 juillet 2021. URL : http://journals.openedition.org/ceb/5582 ; DOI https://doi.org/10.4000/ceb.5582

\section{Ce document a été généré automatiquement le 6 juillet 2021.}

Cahiers balkaniques est mis à disposition selon les termes de la Licence Creative Commons Attribution - Pas d'Utilisation Commerciale 4.0 International. 


\section{Aikaterini Véroni et Evanghélia Paraskévopoulou : deux " grandes actrices » de la scène grecque du XIX siècle}

Aikaterini Veroni and Evangelia Paraskevopoulou, Two Major Actresses of the Greek Scene in the 19th Century

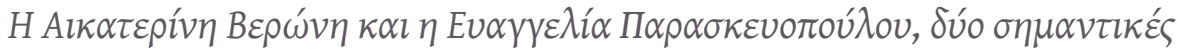

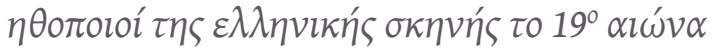

Alexia Altouva

\section{Introduction}

1 Pour aborder la question des élites, je vais examiner comme exemples pertinents les cas de deux grandes actrices et personnalités du théâtre jouissant d'un grand prestige dans la Grèce de la Belle Époque, Aikaterini Véroni (1867-1955) et Evanghélia Paraskévopoulou (1866-1938) qui ont mis en œuvre des tactiques internationales et ont introduit sur la scène théâtrale grecque le vedettisme ${ }^{\mathbf{1}}$.

2 Selon les recherches étendues et récemment achevées sur leur vie et leur carrière, ces deux protagonistes ont contribué de manière décisive à l'épanouissement de plusieurs secteurs du théâtre grec. Entre autres, on peut souligner le développement de l'art dramatique, l'enrichissement du répertoire, la stimulation de l'intérêt concernant l'action théâtrale aussi bien que la formation de l'opinion publique. Leur contribution notoire dans le domaine du théâtre, englobant une série d'innovations apportées dans le statut professionnel de l'artiste, aussi bien que l'influence profonde qu'elles ont exercée sur la classe bourgeoise grecque de l'époque, a conduit à l'adoption de certaines caractéristiques élitistes qu'on va analyser par la suite. 


\section{Secteurs de prédominance du vedettisme}

\section{L'emploi des femmes}

3 Le premier secteur dans lequel elles ont imposé un régime nouveau et pionnier est celui de l'emploi des femmes. Selon la tradition prédominante, en accord avec les données de la société grecque de l'époque, jusqu'à la domination du vedettisme, les femmes participaient à des troupes de caractère familial ou à des troupes plus grandes et mieux organisées, mais sans être toutefois considérées par leurs collègues masculins comme des membres égaux. Elles jouaient plutôt un rôle secondaire ou de complément, et elles étaient obligées d'obéir au pouvoir d'un homme, du chef de la compagnie, le plus souvent un parent, leur père, frère ou mari. C'est pendant la décennie 1890 que les grandes actrices sont arrivées à imposer la présence des femmes dans la vie théâtrale, inaugurant une ère moderne ${ }^{2}$.

Dès 1880, elles avaient déjà effectué de longues carrières à l'étranger, dans le sud-est de l'Europe et le bassin oriental de la Méditerranée ${ }^{3}$, et elles avaient collaboré avec les «acteurs-managers» (actor-managers) les plus importants de l'époque. Parmi eux, on peut citer Démosthène Alexiadis (1839-1916), Dionyssios Tavoularis (1840-1928), Nikolaos Lekatsas (1847-1913) et Démétrios Kotopoulis (1848-1919). Grâce à leur talent et leurs facultés, elles ont acquis d'innombrables partisans, admirateurs ou protecteurs, et cette popularité leur a assuré le pouvoir de participer au processus de prise de décision ${ }^{4}$. Pendant longtemps, elles ont été les facteurs clés dans les troupes théâtrales et parfois, même assez souvent, elles se sont également chargées de l'administration de compagnies entières.

\section{Le nouveau style dramatique}

5 Progressivement, après être devenues des dirigeantes influençant fortement la vie culturelle du pays, elles ont eu accès à l'élite des professionnels du théâtre, et acquis le pouvoir d'éduquer le public et de former son esthétique. Il s'agit du deuxième domaine dans lequel elles ont apporté des réformes importantes. Paraskévopoulou et Véroni ont substantiellement contribué toutes deux à la rénovation de l'art dramatique. Influencées d'une part par le style dramatique que Nicolaos Lekatsas a introduit dans le théâtre grec et adaptant, d'autre part, des aspects différents de l'art sublime de Sarah Bernhardt (1844-1923), chacune des deux protagonistes a élaboré un style personnel, tout à fait unique, qui est devenu un exemple à suivre par les autres actrices de leur génération 5 .

6 Paraskévopoulou s'est distinguée par son style vibrant, comme par l'expression de sentiments intenses et passionnés, tandis que Véroni a promu le profil d'une artiste cultivée, plus modérée et disciplinée. L'une était adorée pour ses poses de statue, l'autre pour son style aristocratique et sa voix mélodieuse; toutes les deux ont été charismatiques et, mises à la tête d'acteurs dévoués à l'art dramatique, elles ont formé un nouveau contexte d'expression artistique ${ }^{6}$.

7 Bien sûr, puisque c'étaient précisément ces vedettes qui décidaient de la sélection du répertoire selon leurs propres facultés, le résultat final touchait toute la compagnie qui devait s'adapter aux conditions nouvelles, sans être toujours capable de le faire. 
Évidemment, un contraste extrême était visible durant la représentation, entre la grande actrice et ses collègues. Dans le cas de Paraskévopoulou, étant elle-même une perfectionniste, elle a voulu éliminer les différences, essayant de contrôler si possible l'évolution de l'action sur scène. Même, elle n'hésitait pas, souvent, à interrompre le spectacle chaque fois qu'elle voulait donner des instructions ou des conseils concernant l'attitude d'un collègue, ou encore à exiger le baisser du rideau au cours de la représentation. Elle était également très compétente dans le domaine de la gestion économique.

\section{L'enrichissement du répertoire}

9 Le troisième secteur auquel elles ont donné une impulsion toute nouvelle est celui de l'enrichissement du répertoire et de la dramaturgie originale. Le vedettisme a été lié aux pièces dites «bien faites " qui ont inondé la scène grecque de la fin du XIX siècle. Les vedettes grecques, s'efforçant de promouvoir leur image de grande actrice, ont reproduit les pièces et les rôles interprétés par les célèbres actrices européennes, Sarah Bernhardt en particulier. De cette façon, elles ont contribué à renforcer la réception de la dramaturgie française contemporaine et à la diffuser en Grèce.

Par ailleurs, elles sont devenues les muses des grands dramaturges du théâtre national qui ont manifesté une créativité intense en cette fin du siècle. L'écrivain le plus important et le plus efficace pour la promotion de leur carrière a été Démétrios Vernardakis (1833-1907) . Personnalité polyvalente, dramatiste, professeur d'université, historien, membre de l'Académie, il appartenait à l'élite des intellectuels grecs et ses décisions, ou préférences exprimées, ont joui d'un grand prestige et pouvaient s'imposer complètement. De ce point de vue, son initiative de collaborer avec "Ménandre ", la troupe théâtrale où Véroni a été protagoniste, et de lui confier le personnage de Fausta (de la pièce homonyme), a été décisive pour la carrière de l'actrice. Il est intéressant de noter que, après la première de Fausta à Athènes en octobre 1893, Véroni a réalisé une tournée très réussie et rentable en Égypte, qu'elle a joué au Palais du Yildiz à Constantinople ${ }^{8}$ et voyagé à Paris aux frais de la communauté grecque d'Alexandrie'.

11 Paraskévopoulou a eu un parcours parallèle. Toutes les deux nées à Constantinople, elles avaient reçu des influences artistiques similaires (l'école de Lekatsas et l'art de Bernhardt), et Paraskevopoulou a été aussi choisie pour jouer un rôle de premier plan dans des événements majeurs. Il s'agit de la représentation d'ÆEdipe qui s'est tenue en 1887 à Athènes et de celle de la Duchesse d'Athènes mise en scène, deux ans plus tard, en 1889, à Philippopolis. Dans le premier cas, l'événement a été organisé par le Rectorat de l'Université d'Athènes à l'occasion du cinquantième anniversaire de sa fondation. La mise en scène était due à Anghelos Vlachos (1838-1920). Paraskévopoulou a été invitée à interpréter le rôle de Jocaste, fait considéré comme ultimement honorable pour la jeune Évanghélia ${ }^{10}$.

12 La Duchesse d'Athènes a été un moment remarquable dans son parcours théâtral. Le fait que l'écrivain de la pièce, Kléon Rangavis (1842-1917), ait été aussi ambassadeur de Grèce dans la région, a ajouté du prestige à l'événement tout aussi bien qu'à la participation de Paraskévopoulou. Une pléiade de personnalités éminentes appartenant aux domaines de la politique, du commerce, de la banque, etc. ont assisté au spectacle. Le résultat a été un triomphe indéniable ${ }^{11}$. Dès lors, la carrière de Paraskévopoulou a 
tourné au vedettisme. Les années suivantes, elle a remporté de grands succès dans les rôles des héroïnes interprétées par la Divine Sarah; on lui donna même le surnom de "Sarah de l'Orient». Elle a réalisé une tournée légendaire en Égypte et elle a par la suite dominé comme « vedette absolue » la scène du théâtre d'Athènes et la conscience de ses spectateurs. Paraskévopoulou est reconnue alors comme une valeur absolue du théâtre et une véritable personnalité de la vie sociale du pays.

On constate donc que toutes les deux, à un moment donné, ont atteint l'élite du monde théâtral et social du pays, grâce à leur talent et au soutien reçu de l'élite intellectuelle de l'époque. Dans une certaine mesure, elles ont été choisies à cet effet. Le climat de concurrence entre les deux vedettes et leurs partisans, atteignant son point culminant en 1893, les a situées au-dessus même des élites, et en tant que personnages emblématiques, les a placées dans la Galerie d'art du théâtre grec de tous les temps ${ }^{12}$.

\section{Séduire les spectateurs}

Il s'agit du quatrième domaine défini par la présence et l'action des vedettes : celui du pouvoir exercé sur le public. La relation entre chaque protagoniste et son public constitue un lien puissant et bidirectionnel. La prédominance du vedettisme dans le théâtre grec de la fin du xix siècle a réussi à provoquer des réactions, dites "sans précédent ", et à causer des changements radicaux dans la mentalité d'une grande partie de la société locale.

Pour la première fois dans la création du théâtre grec moderne, les classes sociales supérieures se sont intéressées à ses progrès. Les personnes qui, dans le passé, avaient systématiquement ignoré l'action des troupes en faveur de l'opéra et des chanteuses lyriques étrangères, se sont tout à coup précipitées pour se procurer un billet et applaudir les protagonistes grecques. En même temps, elles n'ont pas hésité à exprimer leur générosité, en soutenant financièrement le théâtre local ${ }^{13}$.

\section{Les raisons principales dans la formation du phénomène}


joué également un rôle important dans la conservation de ce climat de compétition et sa conséquence, l'émergence du vedettisme.

C'est dans la presse quotidienne et hebdomadaire - journaux et revues plutôt littéraires - que l'on a publié nombre d'informations et de nombreux articles se référant exclusivement à ces deux personnalités. Grâce à ces témoignages précieux, on peut avoir une vue globale sur leur style d'interprétation, leur présence sur scène ou l'état général du théâtre grec de l'époque, décrits par un groupe des personnes fort diverses, chroniqueurs, directeurs des journaux, dramaturges, poètes. Les vedettes sont devenues le centre d'intérêt de toutes les couches sociales, du bas peuple à la haute bourgeoisie. La popularité des grandes actrices renforcée par le pouvoir de la presse a entraîné la création de modèles sociaux.

\section{Conclusion}

21 Le schéma n'est pas nouveau. Cependant, les conditions spécifiques qui l'ont formé lui donnent un caractère unique et nous permettent de faire certaines remarques sur les modes de création d'une élite du théâtre, telle que l'est une vedette. Les exemples donnés déterminent une série de paramètres qui reflètent les questions majeures posées par la société néohellénique et surtout l'élite intellectuelle de la période.

\section{Ce sont :}

- La réforme de l'art dramatique et du théâtre grec: du point de vue artistique, Véroni et Paraskévopoulou ont réussi à rénover les règles - modèles d'expression dramatique -, et à imposer un style qui s'est appuyé sur l'imitation créative de l'art de la Divine Sarah dans son contexte romantique, tout en l'adoptant aux exigences de la réalité grecque. Tout au long de leurs carrières, elles ont interprété aussi efficacement des rôles classiques que d'autres venants de la dramaturgie contemporaine, tant grecque qu'européenne.

- La question de l'émancipation des femmes : Toutes les deux sont devenues des pionnières, prenant des initiatives capitales et participant activement au processus de prise de décisions concernant des questions professionnelles. Elles ont assumé l'administration des troupes théâtrales et elles ont eu leurs propres revenus, ce qui constitue un fait particulièrement important pour une femme de cette période. Leur dynamisme a ouvert des voies aux femmes de leur époque vers une autonomie professionnelle et économique.

- L'affaire de l'identité nationale: Il s'agit ici d'une question majeure pour le monde intellectuel de l'État néohellénique pendant le xIXe siècle, les deux vedettes ont été considérées comme représentantes idéales de l'art grec moderne et capables de diffuser le théâtre national dans tout l'hellénisme disséminé dans l'Orient grécophone, selon le modèle de la Grande Idée. Ces deux actrices ont en effet accompli leur carrière à l'étranger en jouant pour une longue période le rôle de l'ambassadeur du théâtre et celui de propagateur de la culture hellénique dans le bassin de la Méditerranée et de l'Europe sud-orientale. De cette façon, elles ont servi les politiques des gouvernants et les ambitions de la nation.

- L'influence de la politique : Du point de vue politique, le vedettisme a soutenu la diffusion du projet de Charilaos Trikoupis qui prévoyait le renforcement de la classe bourgeoise destinée à stimuler les mécanismes de l'essor économique du pays. Les vedettes à travers leur répertoire ont porté à la scène théâtrale l'air cosmopolite parisien en proposant à la bourgeoisie locale un exemple vivant à imiter. En plus, puisque le vedettisme émergea dans une période de fermentations politiques intenses, il s'est inévitablement lié à des pratiques liées à cette atmosphère. Le point de convergence majeur se détecte surtout dans les 
mécanismes de manipulation du public et dans la formation de partis rivaux. En 1893, deux partis ont dominé la vie politique et sociale de la capitale : la société a été séparée entre deux politiciens, Charilaos Trikoupis (1832-1896) et Theodoros Deligiannis (1820-1905), les figures les plus populaires de la période, et entre deux actrices, Aikaterini Véroni et Evanghélia Paraskévopoulou, les plus célèbres à la fin du siècle.

Enfin, femmes, artistes, professionnelles, innovatrices, pionnières, Aikaterini Véroni et Evanghélia Paraskévopoulou ont réclamé, gagné et mérité le titre de "vedette ». Avant tout, elles ont répondu aux exigences de la société qui, dans une longue période de difficultés économiques et d'agitation intense à l'intérieur et à l'extérieur du pays, recherchait des normes, des réformateurs et des dirigeants capables dans tous les domaines de la vie sociale.

\section{BIBLIOGRAPHIE}

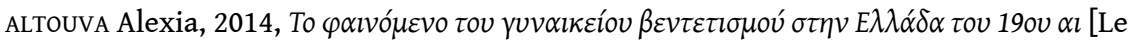
phénomène du vedettisme féminin en Grèce pendant le XIX ${ }^{\mathrm{e}}$ siècle], Athènes : Hérodote.

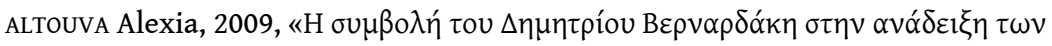

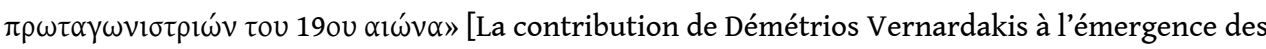

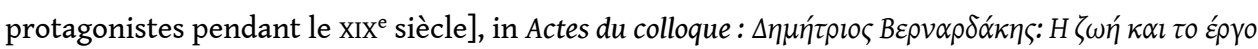

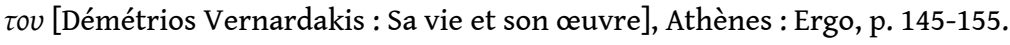

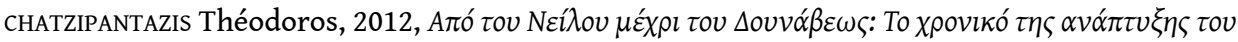

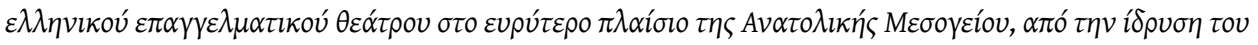

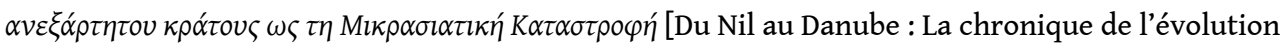
du théâtre professionnel grecque dans la Méditerranée orientale, dès l'établissement de l'État

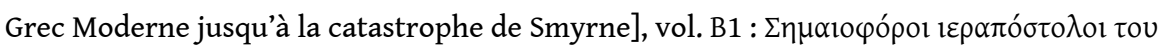

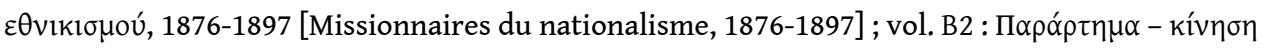
$\varepsilon \lambda \lambda \eta v i \kappa \omega ́ v ~ \theta i \alpha ́ \sigma \omega v, 1876-1897$ [Annexe - Circulation des troupes théâtrales, 1876-1897], Héraklion : Éditions Universitaires de Crète.

CHATZIPANTAZIS Théodoros, 2001, A

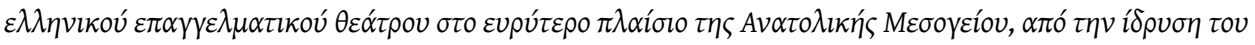

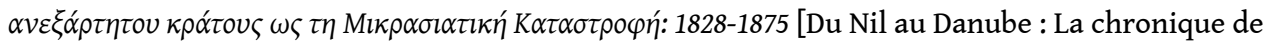
l'évolution du théâtre professionnel grecque dans la Méditerranée orientale, dès l'établissement

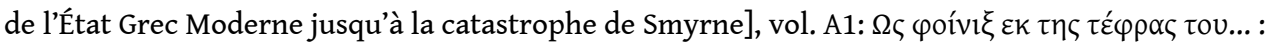

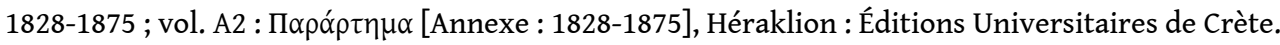
COSER Lewis, 1974, Masters of sociological thought: Ideas in historical and social context, New-York: Harcourt Brace Jovanovich, pp. 396-400.

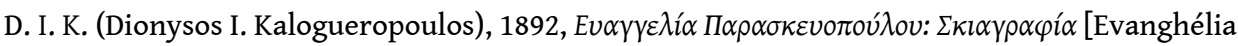
Paraskevopoulou : son Portrait], Athènes : Physis.

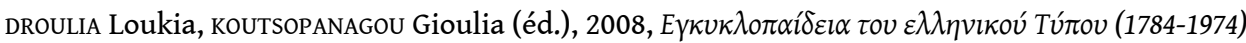
[Encyclopédie de la presse hellénique (1784-1974)], Athènes : INE/EIE. 
GENIEYS William, 2000, « De la théorie à la sociologie des élites en interaction. Vers un néoélitisme? ", in DUCHESNE Sophie, BACHIR M. Cherif, SCHWEYER François-Xavier, et alii, les Méthodes au concret, Paris : P.U.F., p. 81-103.

GLENN Susan, 2000, Female Spectacle: The theatrical roots of modern feminism, Cambridge Mass: Harvard University Press.

Le Figaro, Journal non politique, $1^{\mathrm{er}}$ mai $1895,41^{\mathrm{e}}$ Année, $3^{\mathrm{e}}$ Série, $\mathrm{n}^{\circ} 121$, Paris.

LUCKHURST Mary, MOODY Jane (ed.), 2005, Theatre and Celebrity in Britain, 1660-2000, Basingstoke: Palgrave/MacMillan.

PARETO Vilfredo, 2009, The Rise and Fall of Elites: An Application of Theoretical Sociology, New Brunswick, New Jersey: Transaction Publishers.

PUCHNER Walter, 2012, Hellenophones Theater im Osmanischen Reich (1600-1923), Berlin: Lit Verlag. ROACH Joseph, 2004, «It», Theatre Journal, vol. 56, no 4, pp. 555-568.

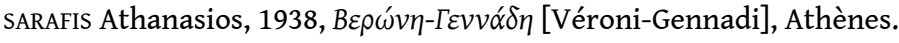

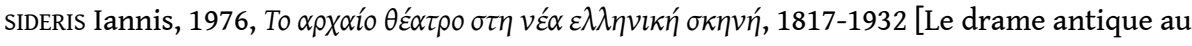
théâtre grec moderne, 1817-1932], Athènes : Ikaros.

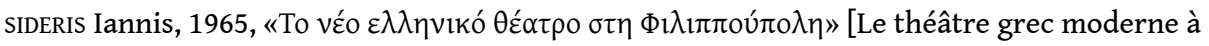
Philippopolis - Plovdiv], Nea Estia, nº 917, 15 septembre 1965, p. 1229-1237.

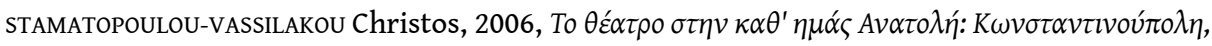
$\Sigma \mu u ́ p v \eta:$ O $\tau \omega \dot{\omega} \mu \varepsilon \lambda \varepsilon \tau \eta \eta_{\mu \alpha \tau \alpha}$ [Le théâtre dans l'Orient grécophone : Constantinople et Smyrne : Huit essais], Athènes : Polytropon.

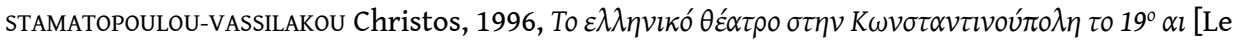

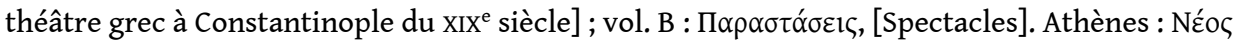

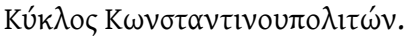

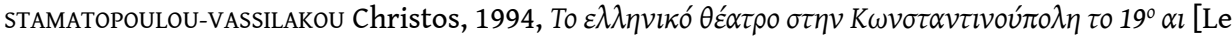

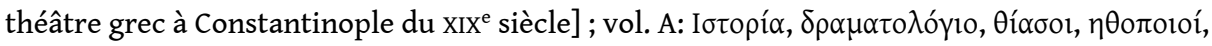

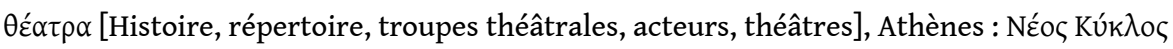

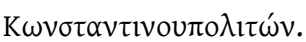

Le Temps, 4 mai $1895,35^{\mathrm{e}}$ Année, $\mathrm{n}^{\circ}$ 1239, Paris.

\section{NOTES}

1. Dans cette communication je reprends les idées principales de ma thèse de doctorat :

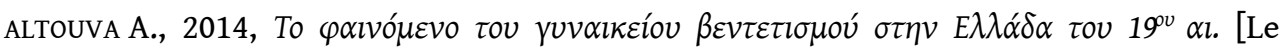
phénomène du vedettisme féminin en Grèce pendant le XIX ${ }^{\mathrm{e}}$ siècle], Hérodote: Athènes.

2. Sur la contribution du théâtre au mouvement féministe, voir GLENN S., 2000.

3. Sur la présence et l'activité des troupes théâtrales grecques dans le sud-est de l'Europe et le bassin oriental de la Méditerranée voir PUCHNER W., 2012 ; STAMATOPOUlOU-VASSILAKOU Chr., 2006 ; idem, 1996 ; id., 1994.

4. Selon la théorie de Vilfredo PARETO « on ne peut parler d'élite qu'à l'intérieur d'une branche d'activités » (GENIEYS W., 2000, p. 85). 
5. Dans la presse quotidienne et hebdomadaire de la période, il y a de nombreux articles qui décrivent le style dramatique des deux actrices grecques. Pour plus d'information sur la presse grecque de cette période voir DROULIA L., KOUTSOPANAGOU G. (éd.), 2008.

6. Pour plus d'information voir altouva A., 2009, p. 145-155.

7. Cf. SARAFIS A., 1938, p. 79.

8. La presse française annonça l'arrivée de la vedette grecque comme suit: "Paris donne depuis hier l'hospitalité à trois artistes dramatiques grecs, M. D. Véroni et Mlles Catherine et Smaragda Véroni, venues pour passer quelque temps parmi nous, et se retremper dans l'atmosphère artistique de nos théâtres. Mlle Catherine Véroni est considérée comme la Sarah Bernhardt grecque et les journaux d'Athènes et de Constantinople ont à plusieurs reprises rendu hommage à son talent extraordinaire et à sa facilité de s'assimiler les rôles les plus difficiles » (Le Figaro, $1^{\mathrm{er}}$ mai 1895, $\mathrm{n}^{\circ} 121$, p. 5).

"Trois artistes dramatiques grecs, M.D. Véroni et Mlles Catherine et Smaragda Véroni, sont venues passer quelque temps à Paris dans le but d'étudier nos théâtres et nos grands artistes. Tous les trois sont des artistes d'un talent supérieur et la presse d'Athènes et de Constantinople a parlé longuement d'eux. C'est surtout Mlle Catherine Véroni, que les Grecs appellent la "Sarah Bernhardt" de l'Orient qui excite l'admiration du public là-bas » (Le Temps, 4 mai 1895, $\mathrm{n}^{\circ} 1239$ : p. 3).

9. Cf. SIDERIS J., 1976, p. 74.

10. Cf. D.I.K., p. 27 ; SIDERIS J., 1965, p. 1233.

11. L'axiome de Pareto concernant la supériorité de certaines personnes grâce à leurs capacités spéciales correspond plutôt aux vedettes de la période (cf. PARETO V., 2009, p. 8, COSER L.-A., 1974, p. 396-400).

12. Pour plus d'information voir ROACH J., 2004, p. 555-568.

13. Sur l'interrelation entre le vedettisme et le facteur de l'économie voir LUCKHURST M., J. MOODY, (éd.), 2005, p. 127-190.

\section{RÉSUMÉS}

L'élitisme au théâtre se relie fortement au phénomène du vedettisme qui a été introduit dans le théâtre grec au XIX siècle par les deux protagonistes, Aikaterini Véroni et Evanghélia Paraskévopoulou. Dans cette communication, on souligne les secteurs différents de prédominance du vedettisme dans lesquels les deux actrices se sont distinguées, l'emploi des femmes, l'adoption d'un nouveau style dramatique, l'enrichissement du répertoire et le pouvoir exercé sur le public. On examine les raisons principales qui ont provoqué la formation de ce phénomène dans le théâtre grec et l'on en tire des conclusions sur la création d'une élite du théâtre, en tenant compte de ce qu'est une vedette à l'époque. Dans ce contexte, on discute aussi d'une série des questions que posent les intellectuels et la société néohellénique de la fin du XIX ${ }^{\mathrm{e}}$ siècle. 
Elitism in theatre has been strongly connected to the phenomenon of celebrities that was introduced in Greek theatre during the 19th century by two protagonists, Aikaterini Veroni and Evangelia Paraskevopoulou. In my intervention I underline the different sectors of predominance of celebrities in which the two actresses had excelled. These are the employment of women, the adoption of a new dramatic style, the enrichment of repertoire, the influence on the audience. I also examine the main reasons that provoked the formation of the phenomenon in Greek theatre and I make some conclusions on the creation of an elite in theatre. In this context is discussed a series of questions set by the intellectuals and Greek society in fin de siècle.

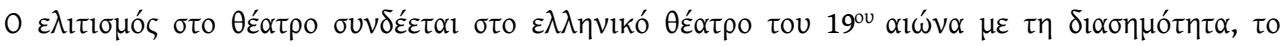

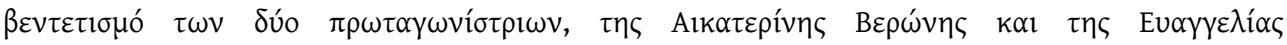

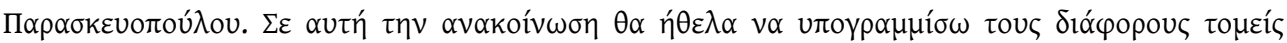

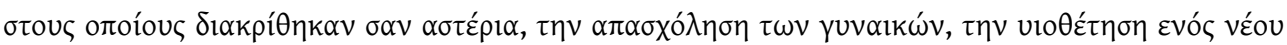

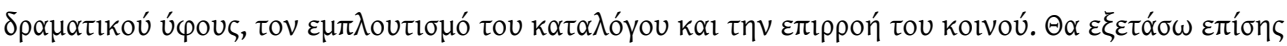

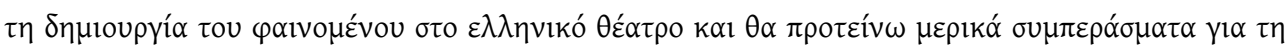

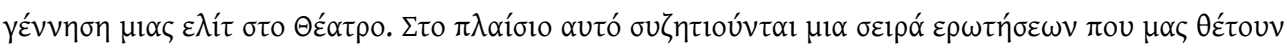

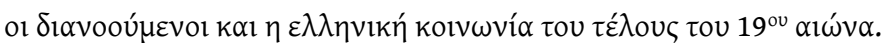

\section{INDEX}

Index géographique : Grèce

Index chronologique : dix-neuvième siècle -- fin

Mots-clés : Véroni Aikaterini (1867-1955), Véroni Aikaterini (1867-1955), Paraskévopoulou Evanghélia (1866-1938), Paraskévopoulou Evanghélia (1866-1938), actrices-vedettes

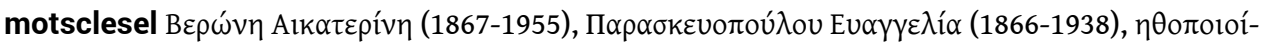

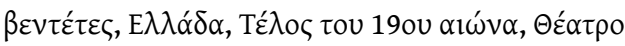
motsclestr Veroni Ekaterini(1867-1955), Paraskevopoulou(1866-1938), Aktrisler- Yıldızlar, Yunanistan, On dokuzuncu yüzyılın sonu, Tiyatro motsclesmk ВЕРОНИ КАТЕРИНИ (1867-1955), ПАРАСКЕВОПУЛУ ЕВАГЕЛЈА (1866-1938), АКТЕРКИТЕ SВЕЗДИ, ГРЦИЈА, КРАЈОТ НА ДЕВЕТНАЕСЕТТИОТ ВЕК, ТЕАТАР

Keywords : Veroni Aikaterini (1867-1955), Actresses-stars, Paraskevopoulou Evanghelia (1866-1938), Greece, End of the nineteenth century, Theatre

Thèmes : Théâtre

\section{AUTEUR}

\section{ALEXIA ALTOUVA}

Département d'études théâtrales, Université d'Athènes 